\title{
Análise de agrupamento e regressão não-linear aplicados ao crescimento in vitro de Leucoagaricus gongylophorus (Singer) Möller em meios de cultura acrescido com diferentes extratos vegetais
}

\author{
Marcelo Dias de Souza ${ }^{1}$ \\ Otávio Peres Filho \\ Sidney Fernando Caldeira ${ }^{2}$ \\ Allan Libanio Pelissari ${ }^{1}$ \\ Alberto Dorval ${ }^{2}$ \\ 'PPG em Ciências Florestais e Ambientais \\ ${ }^{2}$ Departamento de Engenharia Florestal \\ Faculdade de Engenharia Florestal \\ Universidade Federal de Mato Grosso \\ Avenida Fernando Corrêa da Costa, 2367, CEP 78125-070, Cuiabá - MT, Brasil \\ *Autor para correspondência \\ marcelo.dias@florestal.eng.br
}

Submetido em 14/06/2011

Aceito para publicação em 12/09/2011

\section{Resumo}

O estudo teve como objetivo avaliar o crescimento in vitro de Leucoagaricus gongylophorus em meio de cultura acrescido com diferentes extratos de espécies vegetais, por meio da análise estatística multivariada de agrupamento de dados, além de determinar o comportamento do fungo por meio de equações de regressão não lineares. $\mathrm{O}$ fungo foi inoculado em meio BDA acrescido com oito extratos vegetais, além de dois controles (com e sem adição de $20 \mathrm{~mL}$ de água destilada), em placas de Petri. As placas foram mantidas em câmaras climatizadas à temperatura de $25 \pm 1^{\circ} \mathrm{C}$ no escuro, por um período de 42 dias e o crescimento do fungo foi avaliado em intervalos semanais. Foi comprovado que a análise de agrupamento e o modelo de Chapman-Richards são eficientes para verificar a similaridade de diferentes extratos vegetais no desenvolvimento do fungo, bem como estimar e descrever o crescimento de L. gongylophorus.

Palavras-chave: Formigas cortadeiras, Fungo, Modelo matemático, Regressão linear

\section{Abstract}

Clustering and nonlinear regression analysis applied to the in vitro growing of Leucoagaricus gongylophorus (Singer) Möller in a culture environment enriched with different botanical extracts. The study aimed to evaluate the in vitro growing of Leucoagaricus gongylophorus in a culture environment enriched with different extracts from botanical species, via multivariate statistical analysis of data clustering, besides identifying the fungus behavior through nonlinear regression equations. The fungus was inoculated into a BDA 
medium enriched with eight botanical extracts, along with two controls (with and without the addition of $20 \mathrm{~mL}$ distilled water), in Petri plates. The plates were kept in a climatized chamber at a $25 \pm 1^{\circ} \mathrm{C}$ temperature in the dark, during a 42 days period and the fungus growing was evaluated at weekly intervals. It was proved that the clustering analysis and the Chapman-Richards model are efficient to verify the similarity of different botanical extracts in the fungus development, as well as to estimate and describe the growing of L. gongylophorus.

Key words: Fungus, Mathematical model, Leaf-cutting ants, Linear regression

\section{Introdução}

As formigas cortadeiras, pertencentes à Família Formicidae e Tribo Atttini, estão situadas entre as mais graves pragas da agrossilvicultura brasileira, em razão dos danos às culturas agrícolas e florestais. Estes insetos necessitam do material de origem vegetal, o qual serve de substrato para o crescimento do fungo Leucoagaricus gongylophorus (Möller) Singer (Agaricales: Agaricaceae).

Os gêneros de formigas cortadeiras, como Atta e Acromyrmex, dependem primordialmente deste fungo como alimento para o desenvolvimento das larvas (QUINLAN; CHERRETT, 1979; HÖLLDOBLER; WILSON, 1990). As operárias utilizam os materiais vegetais como substrato para o crescimento de seu simbionte e as mesmas complementam sua alimentação com líquidos extraídos das folhas, durante a coleta e processamento desse substrato (BASS; CHERRETT, 1995). A relação simbiótica entre o fungo e as formigas proporciona benefícios a ambos (SCHULTZ et al., 2005), pois estas se beneficiam do fungo por meio da quebra das enzimas, possibilitando a desoxidação de compostos secundários oriundos dos vegetais e que poderiam agir como inseticidas naturais às formigas (NORTH et al., 1999). Já o fungo se beneficia, entre outras razões, pela manutenção do ambiente livre de competidores, por meio da aplicação de compostos antibióticos produzidos pelas formigas (CURRIE et al., 1999).

Segundo Borba et al. (2006), a preferência para o corte das espécies vegetais está relacionada com a exigência nutricional do fungo mutualista, onde em um ecossistema natural, as formigas mostram preferência por algumas espécies vegetais, as quais são constantemente desfolhadas, enquanto outras não são atacadas, embora sejam abundantes e localizadas próximas ao ninho.
Devido à importância que as formigas exercem ao causar danos aos vegetais e pela complexa simbiose com o fungo L. gongylophorus (HEBLING et al., 1994), alguns autores têm realizado trabalhos visando fornecer subsídios sobre inibição ou o beneficiamento no crescimento in vitro do fungo mutualista, porém o comportamento deste complexo é de difícil entendimento, já que apresenta um crescimento lento em laboratório. Alguns autores têm adicionado extratos vegetais em meios de cultura com o objetivo de catalisar o crescimento ou inibir o desenvolvimento in vitro do fungo, pois o fungo cresce eficientemente em componentes encontrados nas folhas das árvores (SIQUEIRA et al., 1998), ou pode ser prejudicados pelos metabólitos secundários (RIBEIRO et al., 1998).

A definição de grupos de tratamentos é útil quando se deseja desenvolver hipóteses relativas à natureza dos dados. A técnica mais comumente utilizada para essa finalidade é a análise de agrupamento, que reúne indivíduos ou objetos mais parecidos uns com os outros em grupos, ao mesmo tempo em que maximiza a heterogeneidade entre os grupos. No entanto, a análise de agrupamento é usada, principalmente, como uma ferramenta exploratória (HAIR JÚNIOR et al., 2005). Assim, a aplicação de técnicas de regressão torna-se importante para a avaliação quantitativa de um conjunto de dados.

A análise de regressão é a técnica de dependência mais amplamente usada e versátil para analisar a relação de uma variável com outra variável preditora (HAIR JÚNIOR et al., 2005), sendo os modelos de regressão linear aplicados nas mais diversas áreas do conhecimento. Apesar de a regressão linear ser amplamente utilizada para a representação dos fenômenos biológicos como o crescimento de organismos vivos na sua fase inicial, quando estudados durante um tempo maior de desenvolvimento do organismo, não é a forma 
matemática mais representativa estatisticamente (REGAZZI, 2010).

Portanto, o presente estudo teve como objetivo avaliar o crescimento in vitro de L. gongylophorus em meio BDA acrescidos com diferentes extratos vegetais com o intuito de melhor estudar o organismo e sua ação interativa no ambiente in vitro, por meio da análise estatística multivariada de agrupamento de dados e descrever esse crescimento por meio de equação de regressão não-linear.

\section{Material e Métodos}

O experimento foi realizado no Laboratório de Patologia Florestal da Faculdade de Engenharia Florestal, Universidade Federal de Mato Grosso (UFMT), campus de Cuiabá. O fungo L. gongylophorus foi coletado em formigueiros de Atta sexdens rubropilosa (saúva-limão) presentes no Campus da UFMT em Cuiabá. Os extratos foram obtidos a partir de folhas de oito espécies florestais (Tabela 1).

TABELA 1: Relação das espécies vegetais coletadas no Campus da UFMT (Cuiabá-MT) usadas na obtenção dos extratos para avaliação do crescimento in vitro de L. gongylophorus em meio BDA. Laboratório de Patologia Florestal, FENF/UFMT, Cuiabá - MT, 2010 .

\begin{tabular}{lll}
\hline Nome vulgar & \multicolumn{1}{c}{ Espécie } & \multicolumn{1}{c}{ Família } \\
\hline Gonçaleiro & $\begin{array}{l}\text { Astronium fraxinifolium } \\
\text { Schott }\end{array}$ & Anacardiaceae \\
Carvãozinho & $\begin{array}{l}\text { Callisthene fasciculata } \\
\text { Mart. }\end{array}$ & Vochysiaceae \\
Jenipapo & Genipa americana L. & Rubiaceae \\
Jatobá & Hymenaea courbaril L. & Leguminosae \\
Seringueira & Hevea brasiliensis M. Arg. & Euphorbiaceae \\
Ingá & Inga edulis Mart. & Fabaceae \\
Pau-terra & Qualea grandiflora Mart. & Voquisiaceae \\
Cambará & Vochysia divergens Pohl. & Vochysiaceae \\
\hline
\end{tabular}

As folhas das espécies selecionadas foram secas em estufa a $40^{\circ} \mathrm{C}$ durante $48 \mathrm{~h}$ e, em seguida, efetuada a moagem do material em liquidificador. Os extratos foram obtidos por meio do processamento da massa seca das folhas com água destilada, na proporção de 1:6 (peso: volume), sendo posteriormente filtrada através de tecido fino ("voil") (SOUZA; VENDRAMIM, 2001), com a transferência imediata de $20 \mathrm{~mL}$ de extrato para $100 \mathrm{~mL}$ de meio de cultura (BORBA et al., 2006; CASTELLANI et al., 2007). Os dois controles consistiram no meio de cultura puro e o segundo com aplicação adicional de $20 \mathrm{~mL}$ a mais de água destilada.

O meio de cultura utilizado foi o BDA preparado com $200 \mathrm{~g}$ de batata, $20 \mathrm{~g}$ de dextrose e $15 \mathrm{~g}$ de ágar para $1000 \mathrm{~mL}$ de água destilada. Os meios foram ajustados para valores próximos a 5,50 utilizando-se soluções de HCL ou NaOH 1mol.L-1 ${ }^{-1}$ Os frascos contendo o meio de cultura e os extratos foram esterilizados em autoclave a $120^{\circ} \mathrm{C}$ e a $1,1 \mathrm{~atm}$ de pressão durante $15 \mathrm{~min}$. Em seguida foram transferidos $15 \mathrm{~mL}$ de meio de cultura para placas de Petri (90mm x 15mm) previamente esterilizadas, onde permaneceram até a completa solidificação.

Para confirmar se o fungo isolado nas placas de Petri era o mesmo cultivado pelas formigas, foram efetuadas observações ao microscópio para comprovar a presença das estruturas denominadas gongilídeos (Figura 1A), que são únicas do fungo L. gongylophorus (CASTELLANI et al., 2007; MIYASHIRA et al., 2010).

Discos de meio de cultura de $5 \mathrm{~mm}$ de diâmetro obtidas com o auxilio de um vazador foram transferidos para as placas de Petri, sendo, posteriormente, seladas com filme de polietileno e incubadas em câmaras climatizadas do tipo BOD à temperatura de $25 \pm 1^{\circ} \mathrm{C}$ no escuro. O crescimento do fungo foi avaliado macroscopicamente com base no diâmetro da colônia, em milímetros, com seis avaliações semanais (BORBA et al., 2006). Foram traçadas duas retas perpendiculares, com ponto de cruzamento coincidindo com o centro do disco do inóculo (Figura 1B), e realizadas as medidas a partir da borda do inóculo até a extremidade do crescimento do fungo, sendo, em seguida, calculado o valor médio dos diâmetros para cada placa.

A análise de agrupamento foi utilizada com o objetivo de agrupar os valores do crescimento do fungo L. gongylophorus relacionados com os efeitos benéficos ou prejudiciais dos extratos. O método utilizado para a sequência de fusão dos agrupamentos foi a ligação média com a distância euclidiana (HAIR JÚNIOR et al., 2005). 

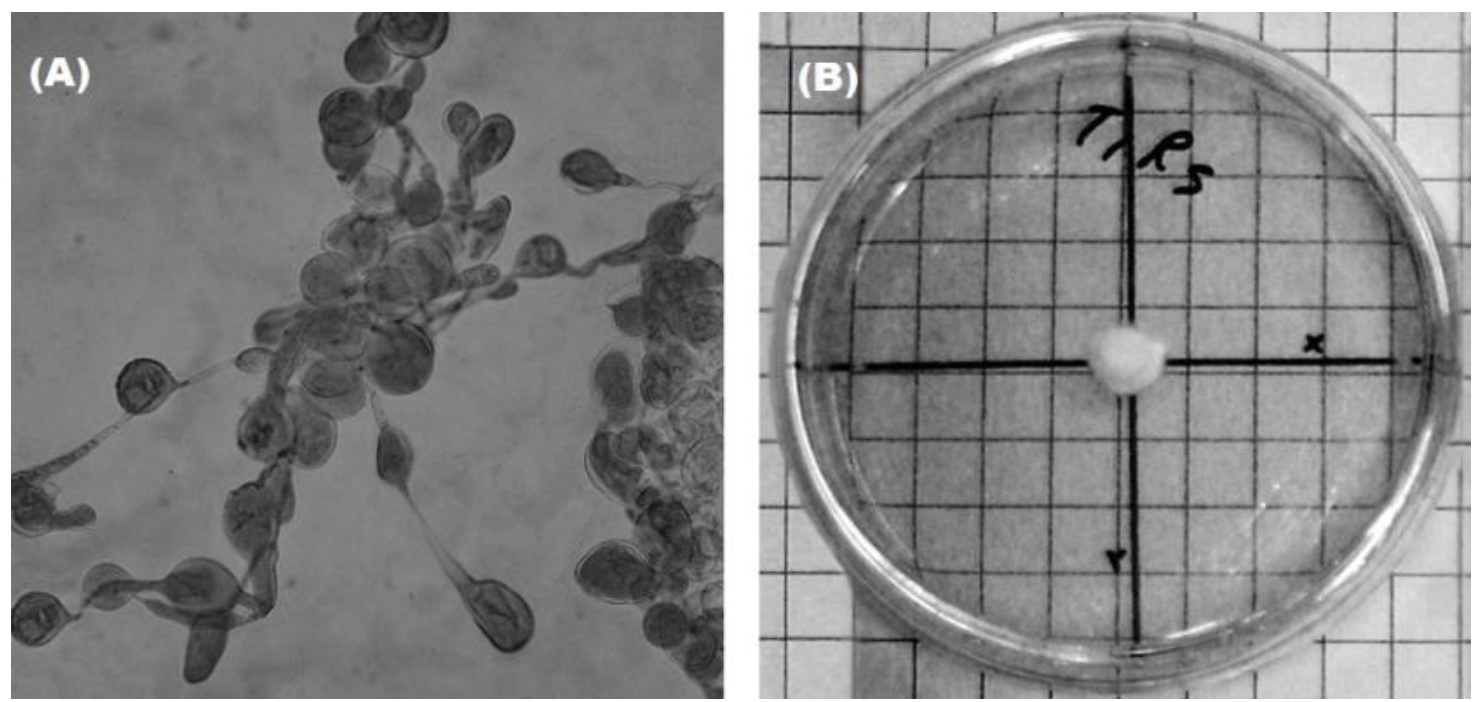

FIGURA 1: Gongilídeos e hifas do fungo L. gongylophorus (A) isolado no laboratório (objetiva de 40x) e placa de Petri com o micélio em desenvolvimento (B). Laboratório de Patologia Florestal, FENF/UFMT, Cuiabá - MT, 2010

Foi adotado como ponto de corte a distância equivalente a $50 \%$ da distância máxima observada, conforme Albuquerque et al. (2006), a fim de facilitar a avaliação e a tomada de decisão sobre os grupos formados.

Para validar os grupos obtidos, foram estimadas e confeccionadas as curvas de crescimento do fungo, sob os diferentes extratos, por meio do ajuste do modelo de regressão não-linear de Chapman-Richards, pois este modelo apresenta maior interpretabilidade em termos biológicos (FLORIANO et al., 2006), sendo expresso por:

$$
d=\beta_{0}\left(1-e^{\left(-\beta_{1} . t\right)}\right)^{\beta_{2}}+\varepsilon_{i}
$$

Em que:

$d=$ diâmetro $(\mathrm{mm})$

$\mathrm{t}=$ tempo (dias)

$\beta_{0}, \beta_{1}$ e $\beta_{2}=$ coeficientes de regressão

$e=$ exponencial

$\varepsilon_{\mathrm{i}}=$ erro associado.

O modelo foi ajustado com o auxílio do pacote estatístico SAS (SAS INSTITUTE, 2008) e o critério de avaliação considerou o menor erro padrão da estimativa em porcentagem $\left(\mathrm{S}_{\mathrm{yx}} \%\right)$ e ao maior coeficiente de determinação ajustado $\left(\mathrm{R}_{\text {aj. }}^{2}\right)$. O $\mathrm{S}_{\mathrm{yx}} \%$ mede a dispersão média entre os valores observados e estimados ao longo da linha de regressão e o $\mathrm{R}_{\text {aj. }}{ }^{\text {. expressa o quanto a }}$ variação total é explicada pela regressão (MACHADO et al., 2002). Também foram avaliados o teste $F$ e a significância dos coeficientes de regressão $(\beta)$ pelo teste $t$, ao nível de $5 \%$ de probabilidade, o qual fornece uma estimativa baseada na probabilidade de que os coeficientes obtidos em muitas amostras de um dado tamanho sejam de fato diferentes de zero. Assim, se não for estatisticamente significativo, a variável independente específica (tempo) não é um bom indicador da variável dependente (diâmetro), devendo, portanto, ser descartada a equação (HAIR JÚNIOR et al., 2005).

\section{Resultados e Discussão}

A adição dos extratos vegetais incorporados ao meio BDA ora favoreceu ora inibiu o desenvolvimento do fungo L. gongylophorus, uma vez que foi observado desenvolvimento micelial diferenciado dos fungos crescendo naquelas condições quando comparados aos controles. Hebling et al. (2000) observaram que componentes das folhas de algumas plantas exercem forte efeito sobre o desenvolvimento do fungo simbionte, beneficiando ou prejudicando o seu desenvolvimento, havendo evidências de que isso ocorra pela presença de metabolitos secundários presentes em algumas espécies vegetais, sendo essa uma das possibilidades para explicar 
as alterações observadas em campo no comportamento de forrageio das formigas (BUENO et al., 1995).

Segundo Siqueira et al. (1998), o fungo simbionte de $A$. sexdens cresce eficientemente em componentes encontrados nas folhas, como o amido e a xilana, sendo estes polissacarídeos importantes fontes de carbono para a dieta das formigas quando metabolizados pelo fungo. Mesmo que a adição de alguns extratos possa beneficiar o desenvolvimento do fungo L. gongylophorus, o mesmo ainda apresentou um crescimento lento em laboratório, pois em todos os tratamentos o fungo não atingiu as extremidades da placa de petri, ao final do experimento (Figura 2), o que corrobora os resultados obtidos por muitos autores que analisaram o desenvolvimento in vitro deste fungo (CAMARGO et al., 2003; LOECK et al., 2004; BORBA et al., 2006; CASTELLANI et al., 2007; 2009).

A análise de agrupamento do diâmetro micelial resultou na formação de cinco grupos ao estabelecer o ponto de corte $(\mathrm{C})$ igual a 0,65 da distância euclidiana, sendo que os grupos apresentaram as seguintes composições: o grupo $1 \mathrm{com}$ as espécies carvãozinho e pau-terra; o grupo 2 com as espécies cambará, ingá, genipapo e seringueira; o grupo 3 composto pelos controles; e finalmente, o gonçaleiro e o jatobá que não apresentaram similaridade com outras espécies, cada um constituindo um grupo isolado (Figura 3).

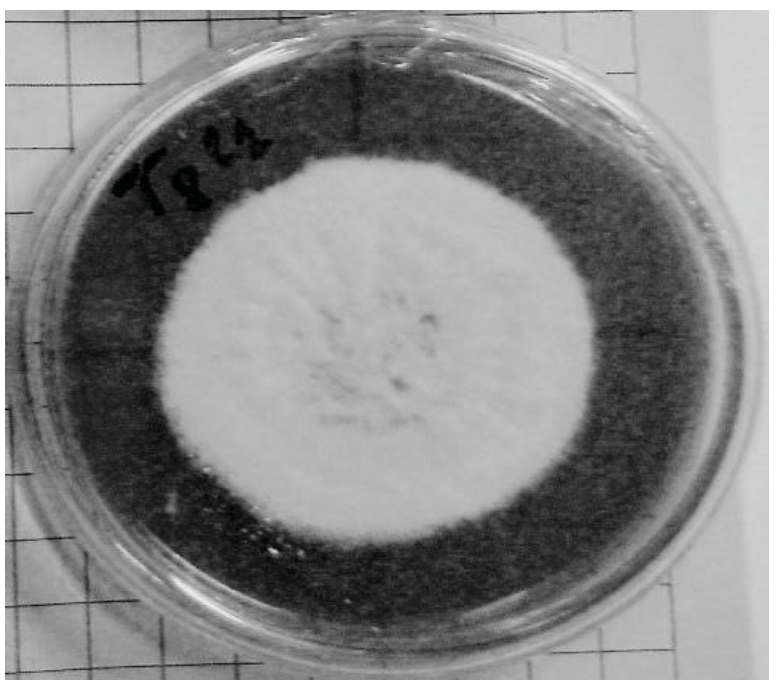

FIGURA 2: Crescimento micelial do fungo L. gongylophorus no meio acrescido com extrato de Hevea brasiliensis no ultimo período de avaliação. Laboratório de Patologia Florestal, FENF/UFMT, Cuiabá - MT, 2010.

O erro padrão da estimativa em porcentagem $\left(\mathrm{S}_{\mathrm{yx}} \%\right)$ é o parâmetro mais eficiente para informar a qualidade dos ajustes de modelos de regressão, pois apresenta o erro médio ao estimar a variável dependente (MACHADO et

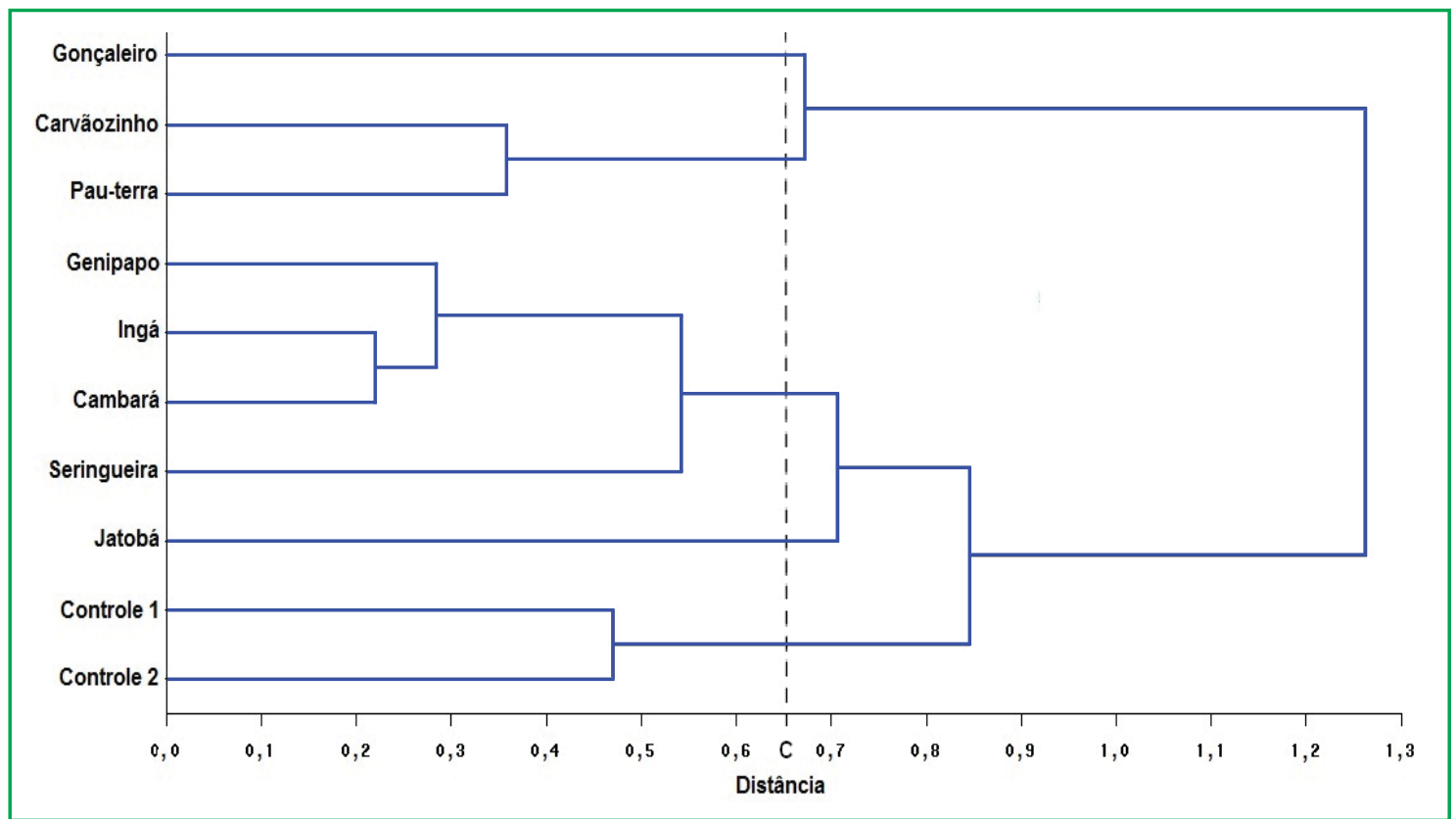

FIGURA 3: Dendrograma da análise de agrupamento do crescimento do fungo L. gongylophorus nos diferentes extratos florestais pelo método da ligação média. Cuiabá - MT, 2010. 
al., 2008). O modelo de Chapman-Richards apresentou bons ajustes (Tabela 2) ao fornecer erros padrão de estimativa $\left(\mathrm{S}_{\mathrm{yx}} \%\right)$ inferiores a $13 \%$, e eficiência na estimativa do crescimento do fungo L. gongylophorus ao apresentar coeficientes de determinação ajustados $\left(\mathrm{R}_{\mathrm{aj}}^{2}{ }^{\cdot}\right)$ superiores a 0,900 , com o menor $\mathrm{S}_{\mathrm{yx}} \%$ e o maior $\mathrm{R}_{\text {aj. }}{ }^{2} \mathrm{de}$ $5,265 \%$ e 0,991 , respectivamente, para o extrato à base de genipapo. Adicionalmente, os valores das estatísticas F e dos coeficientes de regressão ( $\beta$ ) foram significativos, o que demonstra que a variável tempo (dias) é um bom indicador da variável diâmetro $(\mathrm{mm})$ para o estudo do crescimento do fungo L. gongylophorus.

Borba et al. (2006) descreveram o comportamento de crescimento do fungo simbionte de espécies de Acromyrmex spp. por meio de regressão linear e quadrática, onde foi verificado apenas a precisão da curva $\left(\mathrm{R}^{2}\right)$. Porém, cabe ressaltar que a regressão linear não consegue descrever precisamente o crescimento de L. gongylophorus, uma vez que este fungo é de crescimento lento em laboratório, caracterizado por uma fase de crescimento maior em algum determinado intervalo, que com o passar do tempo vai se atenuando até que o processo tende a se estabilizar ou parar, sendo esse processo de avaliação determinado pelo fator tempo em intervalos iguais.

Segundo Godoy et al. (2006), quando o crescimento de alguns organismos vivos for estudado por longos períodos, estes podem não ser satisfatoriamente representados por funções lineares, visto que o comportamento não-linear é o que predomina nos seres vivos, em razão de sua natureza dinâmica e complexa. Para Regazzi (2003), crescimento biológico com variável idade ou tempo são melhores explicados por meio de regressões não-lineares, pois explicam melhor o crescimento do que as regressões lineares.

Silveira et al. (2001), ao compararem a influencia da temperatura no desenvolvimento in vitro de Fusarium verticillioides (Sacc.) Niremberg, Geotrichum candidum Link. ex Pers. e Rhizopus stolonifer (Ehrenb.: Fr.) Vuill., constataram que os modelos matemáticos não lineares explicam melhor o comportamento do crescimento dos referidos fungos. Assim, como observado por Andrade et al. (2005), que ao descreverem o desenvolvimento do fungo Monosporascus cannonballus Pollack \& Uecker no colapso do meloeiro, constataram que os modelos de regressão não-linear foram os mais adequados para explicar o comportamento do referido fungo do que com modelos de regressão linear.

O corte estabelecido na análise de agrupamento foi essencial na explicação do comportamento do crescimento micelial, uma vez que determinou qual dos extratos vegetais influenciaram de forma semelhante às curvas de crescimento do fungo L. gongylophorus (Figura 4).

Os extratos obtidos a partir das folhas das espécies pau-terra e carvãozinho apresentaram comportamentos semelhantes no crescimento do fungo L. gongylophorus ao longo do período de avaliação, com os maiores

TABELA 2: Coeficientes e estatísticas do modelo de Chapman-Richards para a estimativa do crescimento do fungo Leucoagaricus gongylophorus nos diferentes extratos florestais. Cuiabá - MT, 2010.

\begin{tabular}{lcccccc}
\hline \multicolumn{1}{c}{ Extrato } & $\boldsymbol{\beta}_{\mathbf{0}}$ & $\boldsymbol{\beta}_{\mathbf{1}}$ & $\boldsymbol{\beta}_{\mathbf{2}}$ & $\mathbf{S}_{\mathbf{y x}} \mathbf{\%}$ & $\mathbf{R}_{\text {aj. }}^{2}$ & $\mathbf{F}$ \\
\hline Gonçaleiro & $25,5881^{*}$ & $0,1169^{*}$ & $4,3342^{*}$ & 10,761 & 0,957 & $1297,15^{*}$ \\
Carvãozinho & $23,9376^{*}$ & $0,0671^{*}$ & $2,2585^{*}$ & 10,834 & 0,954 & $1263,14^{*}$ \\
Genipapo & $56,2521^{*}$ & $0,0573^{*}$ & $2,4593^{*}$ & 5,265 & 0,991 & $5654,36^{*}$ \\
Jatobá & $42,5376^{*}$ & $0,0872^{*}$ & $4,1310^{*}$ & 7,527 & 0,984 & $2841,21^{*}$ \\
Seringueira & $63,8582^{*}$ & $0,0470^{*}$ & $2,0914^{*}$ & 8,179 & 0,978 & $2309,87^{*}$ \\
Ingá & $59,0342^{*}$ & $0,0533^{*}$ & $2,3334^{*}$ & 6,096 & 0,988 & $1297,15^{*}$ \\
Pau-terra & $17,021^{*}$ & $0,1155^{*}$ & $4,4552^{*}$ & 12,088 & 0,945 & $1023,75^{*}$ \\
Cambará & $69,3521^{*}$ & $0,0377^{*}$ & $1,8545^{*}$ & 6,922 & 0,985 & $3272,66^{*}$ \\
Controle 1 & $32,4501^{*}$ & $0,0720^{*}$ & $1,9640^{*}$ & 5,910 & 0,981 & $4053,58^{*}$ \\
Controle 2 & $30,3623^{*}$ & $0,0717^{*}$ & $2,1782^{*}$ & 7,037 & 0,977 & $2925,53^{*}$ \\
\hline
\end{tabular}

$*$ = significativo ao nível de $5 \%$ de probabilidade. 


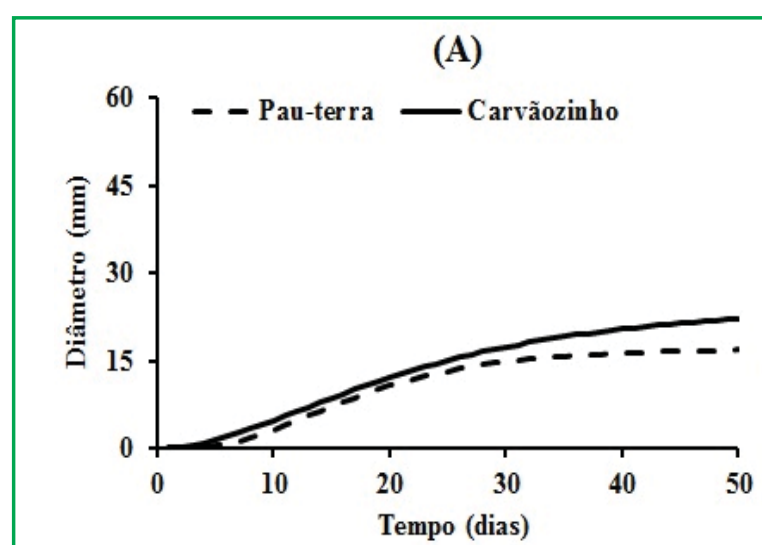

(C)

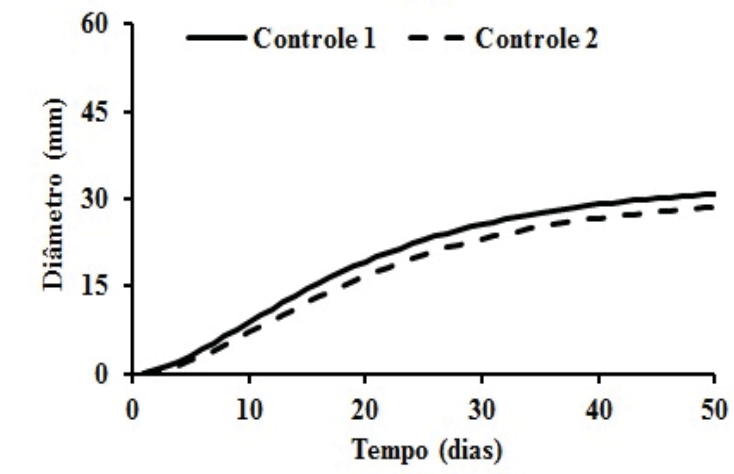

(B)

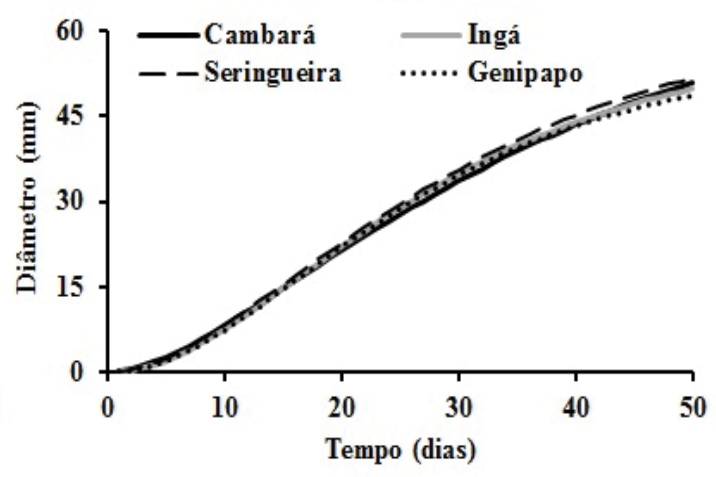

(D)

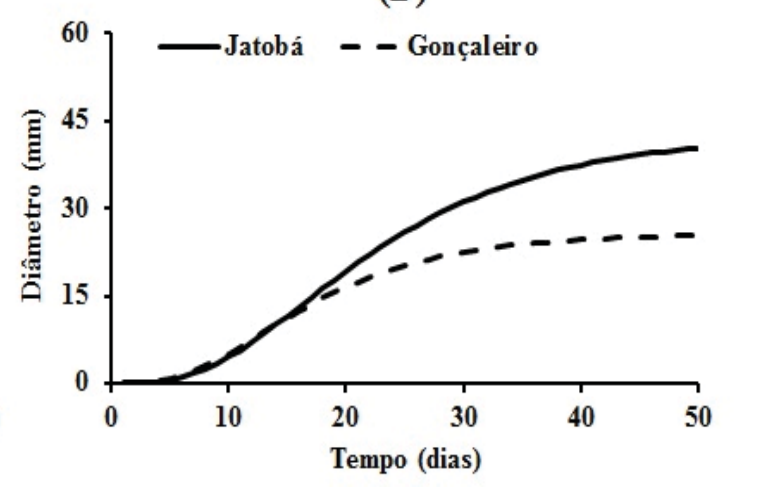

FIGURA 4: Curvas de crescimento do fungo Leucoagaricus gongylophorus em diferentes extratos florestais estimadas pelo modelo de Chapman-Richards. Cuiabá - MT, 2010.

valores no extrato à base de carvãozinho e uma tendência à estabilização da curva de crescimento no extrato pauterra a partir dos 30 dias de cultivo (Figura 4A). Já o grupo formado pelos extratos das espécies cambará, ingá, seringueira e jenipapo proporcionaram os maiores crescimentos em diâmetro, com aproximadamente $50 \mathrm{~mm}$ aos 50 dias, e comportamentos similares entre si (Figura 4B).

Os controles demonstraram tendências semelhantes em função do tempo, com os maiores diâmetros observados no controle 1 , enquanto o controle 2 demonstrou que a adição de $20 \mathrm{~mL}$ de água destilada ao meio de cultura resulta em menor crescimento do fungo $L$. gongylophorus (Figura 4C). Os extratos à base de jatobá e gonçaleiro não resultaram em crescimentos similares a outros tratamentos pela análise de agrupamento. Desta forma, a avaliação comparativa das suas curvas (Figura 4D) demonstrou a dissimilaridade do crescimento de $L$. gongylophorus em função do tempo, com semelhança somente nos períodos iniciais de desenvolvimento do fungo, até aproximadamente 18 dias, e seguidos por maior crescimento no extrato jatobá e forte tendência à estabilização com o gonçaleiro.

O modelo de Chapman-Richards é um dos mais utilizados para modelar o crescimento biológico, devido à grande flexibilidade ao se ajustar aos diversos conjuntos de dados (ZEIDE, 1993), demonstrandose eficiente para avaliar o crescimento do fungo $L$. gongylophorus e comprovar a similaridade observada entre os extratos vegetais na formação dos grupos pela análise de agrupamento.

A partir dos resultados, foi possível concluir que a análise estatística multivariada de agrupamento de dados foi eficiente para discriminar o comportamento do fungo L. gongylophorus nos meios de cultura com a adição dos extratos vegetais, bem como a regressão não-linear, com o modelo de Chapman-Richards pôde descrever e estimar o crescimento desse fungo. 


\section{Referências}

ALBUQUerque, M. A. DE; FERREIRA, R. L. C.; SILVA, J. A. A.; SANTOS, E. S.; STOSIC, B.; SOUZA, A. L. Estabilidade em análise de agrupamento: estudo de caso em ciência florestal. Revista Árvore, Viçosa, v. 30, n. 2, p. 257-265, 2006.

ANDRADE, D. E. G. T.; MICHEREFF, S. J.; BORGES, M. A. S.; ARAÚJO, I. B.; SALES JR, R. Influência da densidade de inóculo e de isolados de Monosporascus cannonballus na severidade do colapso do meloeiro. Summa Phytopathologica, Jaguariúna, v. 31, n. 2, p. 173-180, 2005.

BASS, M.; CHERRETT, J. M. Fungal hyphae as a source of nutrients for the leaf-cutting ant Atta sexdens. Physiological Entomology, London, v. 20, p. 1-6, 1995.

BORBA, R. S.; LOECKI, A. E.; BANDEIRA, J. M.; MORAES, C. L.; CENTENARO E. D. Crescimento do fungo simbionte de formigas cortadeiras do gênero Acromyrmex em meios de cultura com diferentes extratos. Ciência Rural, Santa Maria, v. 36, n. 3, p. 725-730, 2006.

BUENO, O. C., HEBling, M. J. A.; SILVA, O. A.; MATENHAUER, A. M. C. Effect of sesame (Sesamum indicum) on the nest development of Atta sexdens rubropilosa (Hymenoptera: Formicidae). Journal of Applied Entomology, Goettingen, v. 119, p. 341-343, 1995.

CAMARGO, R. S.; FORTI, L. C.; ROCHA, M. M.; MATOS, C. A. O.; LOPES, J. F.; ANDRADE, A. P. P.; VERZA, S. S. The effect of plant diversity on fungus garden development and foraging behavior of leaf-cutting ants (Hymenoptera: Formicidae). Sociobiology, Chico, v. 42, n. 2, p. 1-10, 2003.

CASTELLANI, M. A.; FORTI, L. C.; FENILLE, R. C.; MOREIRA, A.A.; ANDRADE, A. P. P.; NOVCAES, Q. S. Isolation and growth of the symbiotic fungus of Atta capiguara (Hymenoptera: Formicidae). Sociobiology, Chico, v. 50, n. 3, p. 959-972, 2007.

CASTELLANI, M. A.; FORTI, L. C.; FENILLE, R. C.; RAETANO, C. G.; MOREIRA, A. A.; ANDRADE, A. P. P.; CAMARGO, R. S.; LEMOS, R. N. S.; AGUIAR, A. G.; NAGAMOTO, N. S. Growth of the symbiotic fungus of the grass-cutting ant Atta capiguara (Hymenoptera: Formicidae): Effect of grass extracts. Sociobiology, Chico, v. 54, n. 1, p. 283-298, 2009.

CURRIE, C. R.; SCOTT, J. A.; SUMMERBELL, R. C.; MALLOCH, D. Fungus-growing ants use antibiotic-producing bacteria to control garden parasites. Nature, London, v. 398, p.701-704, 1999.

FLORIANO, E. P. MÜLLER, I.; FINGER, C. A. G.; SCHNEIDER, P. R. A. seleção de modelos tradicionais para série temporal de dados de altura das árvores. Ciência Florestal, Santa Maria, v. 16, n. 2, p. 177-199, 2006.

GODOY, M. F.; TAKAKURA, I. T.; CORREA, P. R. Relevância da análise do comportamento dinâmico não-linear (Teoria do Caos) como elemento prognóstico de morbidade e mortalidade em pacientes submetidos à cirurgia de revascularização miocárdica. Arquivos de Ciência da Saúde, Umuarama, v. 12, n. 4, p. 167-71, 2006.

HAIR JÚNIOR, J. F; ANDERSON, R. E.; TATHAM, R. L.; BLACK, W. C. Análise multivariada de dados. 5. ed. Porto Alegre: Bookman, 2005. 597 p.

HEBLING, M. J. A., BUENO, O. C., MAROTI, P. S., PAGNOCCA, F. C.; DA SILVA O. A. Effects of leaves of Ipomoea batatas (Convolvulaceae) on nest development and on respiratory metabolism of leaf-cutting ants Atta sexdens L. (Hymenoptera, Formicidae). Journal of Applied Entomology, Goettingen, v. 124, p. 249-252, 2000.

HEBLING, M. J. A.; BUENO, O. C.; PAGNOCCA, F. C.; SILVA, O. A. A.; FERNANDES, J. B.; VIEIRA, P. C. Derivados de plantas tóxicas como alternativa potencial para o controle de formigas cortadeiras. In: CURSO DE ATUALIZAÇÃO NO CONTROLE DE FORMIGAS CORTADEIRAS, 3, 1994, Piracicaba. Anais... Piracicaba: PCMIP/IPEF, 1994. p. 8-10. Disponível em < http:// www.ipef.br/publicacoes/curso_formigas_cortadeiras/cap03.pdf $>$. HOLLDOBleR, B.; WILSON, E. O. The ants. Cambridge: Harvard University, 1990. 732 p.

LOECK, A. E.; PIEROBOM, C. R.; GUSMÃO, L. G.; AFONSO, A. P. Growth of symbiont fungi of some higher Attini ants in mineral médium. Ciência Rural, Santa Maria, v. 34, n. 1, p. 79-82, 2004.

MACHADO, S. A; CONCEIÇÃO, M. B.; FIGUEIREDO, D. J. Modelagem do volume individual para diferentes idades e regimes de desbaste em plantações de Pinus oocarpa. Ciências Exatas e Naturais, Curitiba, v. 4, n. 2, p.185-196, 2002.

MACHADO, S. doA.; NASCIMENTO, R. G.M.;AUGUSTYNCZIK, A. L. D.; SILVA, L. C. R. da; FIGURA, M. A.; PEREIRA, E. M.; TÉO, S. J. Comportamento da relação hipsométrica de Araucaria angustifolia no capão da Engenharia Florestal da UFPR. Pesquisa Florestal Brasileira, Colombo, n. 56, p. 5-16, 2008.

MIYASHIRA, C. H.; TANIGUSHI, D. G.; GUGLIOTTA, A. M.; SANTOS, D. Y. A. C. Comparison of radial growth rate of the mutualistic fungus of Atta sexdens rubropilosa Forel in two culture media. Brazilian Journal of Microbiology, São Paulo, v. 41, p. 506-511, 2010.

NORTH, R. D.; JACKSON, C. W. HOWSE, P. E. Communication between the fungus garden and workers of the leaf-cutting ant, Atta sexdens rubropilosa, regarding choice of substrate for the fungus. Physiological Entomology, London, v. 24, p. 127-133, 1999.

QUINLAN, R. J.; CHERRETT, J. M. The role of fungus in the diet of the leaf-cutting ant Atta cephalotes. Ecological Entomology, London, v. 4, p. 151-160, 1979.

REGAZZI, A. J. Teste para verificar a igualdade de parâmetros e a identidade de modelos de regressão não-linear. Ceres, Viçosa, v. 50, n. 287, p. 9-26, 2003.

REGAZZI, A. J. Introdução aos modelos de regressão linear e não-linear. Viçosa: UFV, 2010. 157 p.

RIBEIRO, S. B.; PAGNOCCA, F. C.; VICTOR, S. R.; BUENO, O. C.; HEBLING, M. J.; BACCI JR., M.; SILVA, O. A.; FERNANDES, J. B.; VIEIRA, P. C.; SILVA, M. F. G. F. Activity of sesame leaf extracts against the symbiotic fungus of Atta sexdens L. Anais da Sociedade Entomológica do Brasil, Londrina, v. 27, n. 3, p. 421426, 1998.

SAS INSTITUTE. SAS/STAT ${ }^{\circledR} 9.2$ User's Guide Cary: SAS Institute Inc., 2008. 64 p.

SCHULTZ, T. R; MUELLER, U. G.; CURRIE, C. R.; REHNER S. A. Reciprocal illumination: a comparison of agriculture of humans and in fungus-growing ants. In: VEGA, F. (Ed.). Insect-Fungal association: Ecology and Evolution. New York: Oxford University Press, 2005. p. 149-190.

SILVEIRA, N. S. S.; MICHEREFF, S. J.; MARIANO, R. L. R.; TAVARES, L. A.; MAIA, L. C. Influência da temperatura, período 
de molhamento e concentração do inóculo de fungos na incidência de podridões pós-colheita em frutos de tomateiro. Fitopatologia Brasileira, Brasília, v. 26, n. 1, p. 33-38, 2001.

SIQUEIRA, C. G.; BACCI JR.; M. PAGNOCCA, F. C.; BUENO, O. C.; HEBLING, M. J. A. Metabolism of plant polysaccharides by Leucoagaricus gongylophorus, the symbiotic fungus of the leaf-cutting ant Atta sexdens L. Applied and Environmental Microbiology, Washington, v. 64, p. 4820-4822, 1998.

SOUZA, A. P.; VENDRAMIM, J. D. Atividade inseticida de extratos aquosos de meliáceas sobre a mosca-branca Bemisia tabaci (Genn.) Biótipo B (Hemiptera: Aleyrodidae). Neotropical Entomology, Piracicaba, v. 30, n. 1, p. 133-137, 2001.

ZEIDE, B. Analysis of growth equations. Forest Science, Bethesda, v. 30, n. 3, p. 594-616, 1993. 